\title{
Fatores associados à procura por serviços de saúde numa perspectiva relacional de gênero
}

\author{
Factors associated with the demand for health services \\ from a gender-relational perspective
}

Cleice Daiana Levorato ${ }^{1}$

Luane Marques de Mello ${ }^{1}$

Anderson Soares da Silva ${ }^{1}$

Altacílio Aparecido Nunes ${ }^{1}$

${ }^{1}$ Departamento de Medicina Social, Faculdade de Medicina de Ribeirão Preto, Universidade de São Paulo. Av. Bandeirantes 3900,

Monte Alegre. 14.049-900 Ribeirão Preto SP Brasil. altacilio@fmrp.usp.br
Abstract Male culture values involve risk behaviors to health, since the way men perceive and experience their masculinity is one of the most influential shapers of falling sick and dying. The scope of this study was to identify the factors associated with the demand for health services and differences between the sexes by selecting users of health services. The dependent variables were sex of the user (cultural indicator) and failure to seek out health services. Independent variables included socio-demographic and clinical-epidemiological characteristics. The Prevalence Ratio studied by univariate and multivariate analysis was used in the analysis of the association between variables. The factors associated with non-demand for health services included: being male, hours of operation of health care facilities, working hours of the user and not having any disease. By multivariate analysis the factors facilitating demand for health services (protection) were: being female aged 26 to 49 years. Effective consolidation of a health care model that questions the contradiction that exists between the epidemiological data regarding men's health and the position of the health services using common sense regarding the apparent invulnerability of men to falling sick is of great relevance.

Key words Health policies, Gender, Men's health, Health services
Resumo Os valores da cultura masculina envolvem comportamentos de risco à saúde, sendo que a forma como os homens constroem e vivenciam a sua masculinidade torna-se uma das matrizes dos modos de adoecer e morrer. O objetivo deste estudo foi identificar os fatores associados à procura por serviços de saúde e diferenças entre os sexos, selecionando usuários de serviços de saúde, investigando como variável dependente o sexo do usuário (indicador cultural) e a não procura pelo serviço de saúde e variáveis independentes: características sóciodemográficas e clínico-epidemiológicas. Para a análise de associação entre variáveis empregou-se a Razão de Prevalência através de análises univariadas e multivariada. Os fatores associados à não procura por serviços de saúde foram: sexo masculino, horário de funcionamento das unidades de saúde, horário de trabalho do usuário e não possuir nenhuma doença. Pela análise multivariada, os fatores facilitadores da procura por serviços de saúde (proteção) foram: sexo feminino entre 26 e 49 anos de idade. É relevante a efetiva consolidação de um modelo de atenção à saúde que questiona a contradição existente entre os dados epidemiológicos quanto à saúde masculina e a posição dos serviços de saúde de permanecerem no senso comum da invulnerabilidade dos homens ao adoecimento.

Palavras-chave Políticas de saúde, Gênero, Saúde masculina, Serviços de saúde 


\section{Introdução}

O alcance do amplo espectro das relações culturais e sociais, abordado por uma ótica da sociedade e da saúde, demonstra que não só aquelas produzem riscos à saúde, mas também os processos econômicos e políticos, de formas diferentes, fixando assim, a importância da visualização da saúde atrelada a uma estrutura social. Dessa forma, esta focalização incorpora a dimensão de gênero como um determinante do processo saúde-doença dos homens $s^{1-2}$. O contexto cultural brasileiro impõe as marcas de identidade, defendidas como pontos de referência para o reconhecimento do ser homem, como por exemplo: o ser provedor, relacionado ao trabalho e à família; dominador, a quem compete o poder nas relações de gênero e, heterossexual, sendo o interesse sexual por mulheres uma instância julgadora da masculinidade ${ }^{1-4}$. Sublinhando, nesta linha de reflexão, nas relações socioculturais, que homens e mulheres estabelecem, assinala-se que a ideia de cuidados com a saúde é associada à fragilidade, e os serviços de saúde costumam ser identificados como locais de mulheres, crianças e idosos $^{5-7}$.

Estudos ${ }^{8,9}$ sobre as principais causas que levam à internação masculina evidenciaram, em ordem de maior ocorrência, aquelas relacionadas a doenças dos aparelhos respiratório, digestivo e circulatório, infecciosas e lesões externas, sendo que $75 \%$ das enfermidades e agravos na população de homens concentram-se em: cardiologia, urologia, saúde mental, gastroenterologia e pneumologia. Dentre os cânceres mais comuns em homens de acordo com o Instituto Nacional do Câncer (INCA), a neoplasia maligna de próstata, com incidência em 2010 de 52 mil $\operatorname{casos}^{10}$, todavia, de acordo com a Sociedade Brasileira de Urologia ${ }^{11}$, analisando 1.061 homens, na faixa etária de 40 a 70 anos, identificou-se que apenas $32 \%$ realizaram o exame de toque retal, apesar de $76 \%$ referirem que possuem o conhecimento de que ele é realizado para prevenção desse tipo de câncer. Dados de 2007 informam que $60 \%$ das principais causas de internações de homens pelo SUS foram associadas a problemas coronarianos e hipertensão $\operatorname{arterial}^{12}$.

Quanto à influência da postura comportamental masculina as mortes ocasionadas devido ao uso abusivo de álcool e situações derivadas, como acidentes de trânsito, quedas e afogamentos, totalizaram entre os anos de 2000 a 2006, $89 \%$, enquanto que as mesmas ocorrências com mulheres foram de apenas $11 \%{ }^{13}$. Focalizando os acidentes de trânsito, verificou-se que, em $2006^{14}, 82 \%$ ocorreram com homens, adultos jovens e residentes em municípios de pequeno porte. Quanto ao uso do tabaco, embora pesquisas datadas de 2008 demonstrem queda no consumo em todas as faixas etárias, sua presença ainda é maior no sexo masculino (19\%), que no sexo feminino $(12 \%)^{15}$. Em referência ao sedentarismo, o número de homens fisicamente inativos correspondeu em $2008^{16}$ a $29,5 \%$ contra $23,5 \%$ de mulheres, demonstrando diferença tênue, todavia existente e que merece salientar.

O comportamento sexual masculino revela, segundo estimativas divulgadas referentes a 2008, que $21 \%$ dos homens, mesmo possuindo relacionamento estável, tiveram, durante o período, relações sexuais casuais e, desses, $63 \%$ não adotaram práticas preventivas como o uso de preservativos ${ }^{17}$. Como se depreende, pode haver aumento de incidência de doenças sexualmente transmissíveis no sexo masculino, propiciando seu aumento também no sexo feminino, o que demonstra como há urgência em se tratar da saúde masculina, visto que esta se coaduna com outros segmentos.

Baseados nas informações da Política Nacional de Atenção Integral à Saúde do Homem, lançada pelo Ministério da saúde do Brasil ${ }^{9}$, e que serviu como marco teórico de referência nesta introdução, o objetivo do presente estudo foi identificar os fatores associados às diferenças pela procura por serviços de saúde entre homens e mulheres, considerando aspectos sociais, econômicos e culturais.

\section{Métodos}

Trata-se de um estudo transversal, com realização de 320 entrevistas individuais, utilizando-se um questionário estruturado e padronizado, aplicado entre os selecionados nos seguintes serviços de saúde do município de Ribeirão Preto (SP): Núcleos de Saúde da Família (NSF) I e IV da Faculdade de Medicina de Ribeirão Preto da Universidade de São Paulo (FMRP/USP) e ambulatórios/enfermarias do Hospital Estadual de Ribeirão Preto (HERP) vinculado à FMRP/USP, visando compreender as possíveis diferenças de procura pelos serviços de saúde, em um contexto sociocultural, levando-se em consideração aspectos relacionais de sexo e gênero. A população de estudo, apesar de abranger mulheres, foca-se nos homens, levando-se em conta o reconhecido diferencial de gênero, como se observa nos pos- 
tulados teóricos, com relação ao adoecimento, à percepção de saúde e à utilização dos serviços ${ }^{1-7}$. Os participantes foram escolhidos aleatoriamente ao se encontrarem nos estabelecimentos de saúde no momento da coleta de dados. As entrevistas foram realizadas nos períodos matutino e vespertino, entre janeiro e maio de 2011. Nos NSF foram entrevistados os usuários que chegavam para atendimento multiprofissional e no HERP os que aguardavam atendimento ambulatorial ou encontravam-se hospitalizados.

As variáveis dependentes foram: sexo do usuário (indicador cultural) e procura pelo serviço de saúde (indicador do uso de serviços de saúde). As variáveis independentes incluíram: características sociais e demográficas, formando um conjunto de 19 variáveis e; características clínicoepidemiológicas, constituídas de 22 variáveis.

\section{Critérios de inclusão e elegibilidade}

Foram selecionados usuários de ambos os sexos na faixa etária de 18 a 65 anos, capazes de se comunicarem verbalmente e compreenderem as perguntas colocadas, que concordaram em participar do estudo. Não foi adotado como critério de inclusão o tipo de cuidado procurado no momento da pesquisa, considerando-se todos os cuidados ofertados, ou seja, atendimentos multiprofissionais.

\section{Critérios de exclusão e inelegibilidade}

Os idosos acima de 65 anos não foram elegíveis, pois, considerando-se que a maior idade geralmente está associada a maior acometimento por doenças crônicas, possivelmente haveria maior procura pelo serviço de saúde. Também não foram elegíveis, mulheres grávidas e aquelas no puerpério, partindo-se do pressuposto de que elas procuram o serviço de saúde com maior frequência ou por motivos relacionados à criança.

\section{Tamanho Amostral}

Levando-se em consideração, o tipo de estudo, uma população 20.000 pessoas dentro da faixa etária escolhida na área de abrangência das Unidades de Saúde incluídas e que a frequência de homens atendidos nas unidades de saúde de Ribeirão Preto (SP), de acordo com Nunes et al. ${ }^{18}$, em pesquisa realizada em 2009, foi de 33\%, a amostra considerada adequada e representativa foi de 320 a 400 pessoas, com um nível de confiança de $95 \%$ e tolerância de erro amostral de 5\%.

\section{Análise estatística}

Na comparação de diferenças variáveis categóricas foi empregado o teste do qui-quadrado, enquanto que, para comparar médias foi empregado o t de Student para amostras independentes. A Razão de Prevalência (RP) e seu Intervalo de Confiança a 95\% foram empregados como medidas de associação em análises univariadas e multivariada por regressão logística. $\mathrm{O}$ nível de significância adotado foi de 5\%.

\section{Considerações éticas}

O Protocolo de Pesquisa foi aprovado pelo Comitê de Ética em Pesquisa do Centro de Saúde Escola da Faculdade de Medicina de Ribeirão Preto da Universidade de São Paulo.

\section{Resultados}

Analisando-se todo o grupo de entrevistados, apesar de haver predomínio do sexo feminino, não houve diferença entre os sexos quanto à sua prevalência $(\mathrm{p}>0,05)$. Com base na faixa etária, verificou-se predominância de pessoas de 26 a 49 anos de idade, seguida pelo grupo de 50 a 65 anos, não se observando predominância de algum sexo quando se comparou a faixa de 18 a 25 anos com a de 26 a 49 anos, assim como esta com a de 50 a 65 anos ( $p>0,05)$, todavia houve diferenças de prevalência do sexo feminino entre a faixa etária de 18 a 25 anos e a de 50-65 anos ( p < 0,05). A Tabela 1 descreve a distribuição das principais variáveis correspondentes aos perfis social e demográfico.

A Tabela 2 apresenta a distribuição das principais variáveis clínico-epidemiológicas dos entrevistados, os quais, quando indagados se possuíam alguma doença, 205 responderam negativamente e 115 positivamente. Quando questionados se procuram algum serviço de saúde regularmente, 191 responderam que sim e 129 que não. Dos que referiram procurar, a utilização se deveu principalmente para a realização de exames, os quais somaram 159 casos, seguidos de 32 procuras devido a tratamento médico contínuo. Dos que não o utilizavam, 50 responderam que não o consideravam necessário, 28 relataram dificuldades para essa prática devido ao horário de funcionamento dos serviços de saúde, 15 referiram se automedicar.

Quanto à existência ou não de serviço de saúde próximo de sua residência, 303 responderam 
Tabela 1. Distribuição das variáveis

sócio-demográficas dos pacientes selecionados nas Unidades de Saúde estudadas, Ribeirão Preto, SP, Brasil.

\begin{tabular}{|c|c|c|}
\hline Variáveis & $\mathbf{N}$ & $(\%)$ \\
\hline \multicolumn{3}{|l|}{ Serviço de Saúde } \\
\hline Núcleos de Saúde da Família I e IV & 250 & $(78,2)$ \\
\hline Hospital Estadual de Ribeirão Preto & 70 & $(21,8$ \\
\hline \multicolumn{3}{|l|}{ Cidade de origem } \\
\hline Ribeirão Preto & 285 & $(89,1)$ \\
\hline Outras & 35 & $(11,9$ \\
\hline \multicolumn{3}{|l|}{ Sexo } \\
\hline Feminino & 181 & $(56,6)$ \\
\hline Masculino & 139 & $(43,4)$ \\
\hline \multicolumn{3}{|l|}{ Faixa etária } \\
\hline 18-25 anos & 52 & $(16,3)$ \\
\hline 26-49 anos & 179 & $(55,9$ \\
\hline $50-65$ anos & 89 & $(27,8$ \\
\hline \multicolumn{3}{|l|}{ Estado civil } \\
\hline Solteiro & 101 & $(31,5$ \\
\hline Casado & 174 & $(54,4)$ \\
\hline Separado & 29 & $(9,1)$ \\
\hline Viúvo & 16 & $(5,0)$ \\
\hline \multicolumn{3}{|l|}{ Grau de instrução } \\
\hline Não possui & 13 & $(4,1)$ \\
\hline Fundamental incompleto & 125 & $(39,1)$ \\
\hline Fundamental completo & 54 & $(16,9$ \\
\hline Médio incompleto & 32 & $(10,0)$ \\
\hline Médio completo & 70 & $(21,9$ \\
\hline Superior Incompleto & 10 & $(3,1)$ \\
\hline Superior completo & 16 & $(5,0)$ \\
\hline \multicolumn{3}{|l|}{ Renda familiar } \\
\hline Inferior a $01 \mathrm{~S} . \mathrm{M}$. & 15 & $(4,7)$ \\
\hline 01-02 S. M. & 142 & $(44,2)$ \\
\hline 03-04 S. M. & 119 & $(37,3)$ \\
\hline Acima de 04 S. M. & 44 & $(13,8$ \\
\hline \multicolumn{3}{|l|}{ Habitação } \\
\hline Própria ou financiada & 233 & $(72,8$ \\
\hline Alugada & 55 & $(17,2)$ \\
\hline Cedida & 32 & $(10,0)$ \\
\hline \multicolumn{3}{|l|}{ Ocupação } \\
\hline Auxiliar de serviços gerais & 20 & $(5,7)$ \\
\hline Do lar & 59 & $(18,6$ \\
\hline Trabalhador rural & 3 & $(0,9)$ \\
\hline Vendedor & 15 & $(4,7)$ \\
\hline Outra & 223 & $(70,1)$ \\
\hline \multicolumn{3}{|l|}{ Situação trabalhista } \\
\hline Autônomo & 75 & $(23,4)$ \\
\hline Celetista & 126 & $(39,3)$ \\
\hline Aposentado/pensionista & 34 & $(10,6)$ \\
\hline Desempregado & 14 & $(4,3)$ \\
\hline Outros & 71 & $(22,1)$ \\
\hline \multicolumn{3}{|l|}{ Horário de trabalho } \\
\hline Manhã & 5 & $(2,5$ \\
\hline Tarde & 5 & $(2,5)$ \\
\hline Noite & 8 & $(3,9)$ \\
\hline Manhã/Tarde & 184 & $(91,2)$ \\
\hline
\end{tabular}

que sim e 17 identificaram que não. Dos que o referiram positivamente, 297 utilizam esse serviço e 23 não. Questionados sobre a última vez que procuraram algum serviço de saúde, 117 identi-

Tabela 2. Distribuição das variáveis clínico-epidemiológicas dos pacientes selecionados nas Unidades de Saúde estudadas, Ribeirão Preto, SP, Brasil.

\begin{tabular}{|c|c|c|}
\hline Variáveis & $\mathbf{N}$ & $(\%)$ \\
\hline \multicolumn{3}{|l|}{ Possui alguma doença } \\
\hline Não & 205 & $(64,1)$ \\
\hline Sim & 115 & $(35,9)$ \\
\hline \multicolumn{3}{|c|}{ Procura o Serviço de Saúde regularmente } \\
\hline Não & 129 & $(40,3)$ \\
\hline Sim & 191 & $(59,7)$ \\
\hline \multicolumn{3}{|l|}{ Por que utiliza o Serviço de Saúde } \\
\hline Exames & 159 & $(83,2)$ \\
\hline Tratamento contínuo & 32 & $(16,8)$ \\
\hline \multicolumn{3}{|l|}{ Não utiliza o Serviço de Saúde } \\
\hline Não considera necessário & 50 & $(38,7)$ \\
\hline Não precisa, pois já cuida da saúde & 7 & $(5,8)$ \\
\hline Ausência de tempo & 4 & $(3,3)$ \\
\hline Não gosta & 5 & $(4,1)$ \\
\hline Restrições alimentares & 1 & $(0,8)$ \\
\hline Acomodação & 3 & $(2,5)$ \\
\hline $\begin{array}{l}\text { Horário de funcionamento dos } \\
\text { serviços de saúde }\end{array}$ & 28 & $(21,7)$ \\
\hline Atendimento demorado & 8 & $(6,6)$ \\
\hline Medo do diagnóstico & 4 & $(3,3)$ \\
\hline Vergonha de expor os sintomas & 3 & $(2,5)$ \\
\hline $\begin{array}{l}\text { Ausência de vínculo com o serviço } \\
\text { de saúde }\end{array}$ & 1 & $(0,8)$ \\
\hline Automedicação & 15 & $(11,6)$ \\
\hline \multicolumn{3}{|l|}{ Serviço de Saúde próximo } \\
\hline Não & 17 & $(5,3)$ \\
\hline Sim & 303 & $(94,7)$ \\
\hline \multirow{2}{*}{\multicolumn{3}{|c|}{$\begin{array}{l}\text { Última vez que procurou o Serviço de } \\
\text { Saúde }\end{array}$}} \\
\hline & & \\
\hline Menos de 01 mês & 86 & $(27,1)$ \\
\hline 01 mês & 64 & $(20,2)$ \\
\hline 06 meses & 117 & $(36,9)$ \\
\hline 01 ano & 41 & $(12,0)$ \\
\hline Mais de 02 anos & 12 & $(3,8)$ \\
\hline \multicolumn{3}{|l|}{ Motivo da procura } \\
\hline Dor & 87 & $(27,2)$ \\
\hline Doença & 92 & $(28,7)$ \\
\hline Tratamento médico & 12 & $(3,8)$ \\
\hline Outro & 129 & $(40,3)$ \\
\hline \multicolumn{3}{|l|}{$\begin{array}{l}\text { Nível de assistência do serviço } \\
\text { procurado }\end{array}$} \\
\hline Primário & 264 & $(82,4)$ \\
\hline Secundário & 40 & $(12,6)$ \\
\hline Terciário & 16 & $(5,0)$ \\
\hline \multicolumn{3}{|l|}{ Possui plano de saúde } \\
\hline Não & 293 & $(91,6)$ \\
\hline Sim & 27 & $(8,4)$ \\
\hline
\end{tabular}


ficaram que há cerca de seis meses, 86 a menos de um mês, 64 há um mês, 41 há um ano e 12 há mais de dois anos. O motivo da última procura revelou que 87 casos foram devido a dores, 92 por doenças, 12 voltados para tratamento médico contínuo e 129 referentes a outros motivos. O nível de serviço procurado somou 264 voltados para atenção primária e 56 no nível secundário.

Os resultados das análises univariadas comparando-se as características sociodemográficas e clínico-epidemiológicas com a não procura pelos serviços de saúde podem ser visualizados na Tabela 3.

$\mathrm{Na}$ Tabela 4 encontram-se os resultados das análises univariadas na comparação das variáveis sociodemográficas e clínico-epidemiológicas com a variável (resposta) sexo. Cidade de origem, faixa etária e estado civil apresentaram associações significativas com o sexo feminino. Assim como o fato de possuir alguma doença e a proximidade de um serviço de saúde à sua residência. As demais variáveis não revelaram associações significativas.

A Tabela 5 apresenta os resultados do efeito independente de cada uma das variáveis na análise multivariada por regressão logística sobre a procura pelo serviço de saúde como variável resposta, observando-se associações positivas com sexo feminino em comparação ao masculino e à faixa etária de 26 a 49 anos entre o sexo feminino.

\section{Discussão}

Nesta sessão, discutem-se os resultados apresentados anteriormente, buscando-se delinear as inferências causais e estatísticas extraídas com base na fundamentação teórica sintetizada a partir da revisão da literatura, a qual propiciou sustentação aos argumentos. Na análise univariada, a prevalência de não procura pelo serviço de saúde foi $35 \%$ menor entre os usuários dos NSF em relação aos do HERP, sendo significativa quando se observa que ser usuário da atenção primária de saúde (APS) é um fator protetor. A categoria referente ao estabelecimento de saúde solicita destaque, recordando-se que a APS, enquanto porta de entrada do sistema de saúde, é importante reguladora da utilização de recursos de alta densidade tecnológica, presente nos demais níveis hierárquicos. Assim, a utilização e o consumo indiscriminado das tecnologias densas, podem também ser regulados por essa porta de entrada, sobretudo, se ela for resolutiva e com boa acessibilidade. Nessa mesma ótica, a Estratégia Saúde da Família (ESF) oportunizou a imple- mentação dos princípios e diretrizes da Atenção Básica e o primeiro contato preferencial para os usuários do SUS ${ }^{19}$.

Observou-se, no presente estudo, que as mulheres buscaram os serviços de saúde 1,9 vezes mais em relação aos homens. A associação observada entre o sexo e a procura por serviço de saúde, vai ao encontro de muitos estudos publicados $^{7,17,20}$, uma vez que ser do sexo feminino foi um fator preditor de maior busca por assistência à saúde, sendo mensurado com magnitude de 2,43 vezes em relação ao sexo masculino. Os dados do $\mathrm{IBGE}^{21}$ revelaram que, em 2009, havia para cada 100 mulheres, 95 homens; essa proporção vem declinando ao longo do tempo em virtude da maior mortalidade masculina. Estudos mostram que, no caso brasileiro, as mulheres utilizam mais os serviços de saúde do que os homens ${ }^{20,22}$, sendo que tais diferenças são determinantes do consumo pelos serviços entre os sexos.

Os participantes desta pesquisa, pertencentes às faixas etárias, compreendidas nos intervalos entre 18 a 25 anos e 26 a 49 anos, sem estratificação por sexo, apresentaram prevalência de 2,42 e 2,57 vezes, respectivamente, em não procurarem o serviço de saúde, logo, situar-se em tais grupos de idade (independente do sexo) é um fator de risco. Estudo conduzido no Brasil ${ }^{23}$ com população semelhante à do presente estudo (adultos e idosos), mostrou diferenças na procura pelo serviço de saúde segundo a idade. Neste, a faixa etária idosa apresentou associação positiva para a maior procura enquanto que as demais apresentaram associação menor. Ainda que a população idosa procure mais os serviços de saúde, quando estratificada por sexo, encontra-se a questão permanente de gênero, mesmo em uma idade cuja necessidade de cuidados com a saúde, geralmente, é mais elevada. Percebendo a idade como uma característica individual, que pode influenciar na procura pelo serviço de saúde, adotou-se como referência a faixa etária considerada como adultos jovens, seguida dos idosos. Costa ${ }^{24}$ expressa que os idosos possuem alta predominância no uso dos serviços de saúde, fato este explicado pelas doenças crônicas decorrentes do envelhecimento ${ }^{25}$.

A associação entre estado conjugal (civil) e sexo (variável resposta) mostrou prevalência 28\% menor de mulheres casadas em relação aos homens. Isto pode ser entendido sob a ótica de gênero e cultura do casamento, da construção do lar, do reflexo social que o ser casado engendra, ou seja, de manutenção das relações sociais de 
Tabela 3. Análises univariadas utilizando-se como estimador de associação a Razão de Prevalência (RP) sendo a variável resposta, a não procura pelo Serviço de Saúde, Ribeirão Preto, SP, Brasil.

\begin{tabular}{|c|c|c|}
\hline Variáveis & $\mathbf{R P}$ & IC95\% \\
\hline \multicolumn{3}{|l|}{ Serviço de Saúde } \\
\hline NSF x Hospital & 0,65 & $(0,50-0,84)^{*}$ \\
\hline \multicolumn{3}{|l|}{ Sexo } \\
\hline Masculino x Feminino & 2,43 & $(1,82-3,42)^{*}$ \\
\hline \multicolumn{3}{|l|}{ Faixa etária (independente do sexo) } \\
\hline $18-25$ anos & 2,42 & $(1,44-4,06)^{*}$ \\
\hline $26-49$ anos & 2,57 & $(1,64-4,05)^{*}$ \\
\hline $50-65$ anos & 1,0 (Ref.) & - \\
\hline \multicolumn{3}{|l|}{ Renda familiar } \\
\hline Inferior a $01 \mathrm{~S} . \mathrm{M}$. & 1,32 & $(0,78-2,23)$ \\
\hline 01- 02 S. M. & 0,39 & $(0,29-0,53)^{*}$ \\
\hline 03-04 S. M. & 0,98 & $(0,67-1,43)$ \\
\hline Acima de 04 S. M & 1,00 (Ref.) & - \\
\hline \multicolumn{3}{|l|}{ Habitação } \\
\hline Própria & 0,38 & $(0,32-0,45)^{*}$ \\
\hline Alugada & 0,40 & $(0,29-0,55)^{*}$ \\
\hline Cedida & 1,00 (Ref.) & - \\
\hline Financiada & 0,53 & $(0,38-0,74)^{*}$ \\
\hline \multicolumn{3}{|l|}{ Ocupação } \\
\hline Auxiliar de serviços gerais & 0,33 & $(0,17-0,64)^{*}$ \\
\hline Do lar & 0,15 & $(0,08-0,28)^{*}$ \\
\hline Trabalhador rural & 1,00 (Ref.) & - \\
\hline Vendedor & 0,60 & $(0,40-0,91)^{*}$ \\
\hline Outra & 0,46 & $(0,40-0,53)^{*}$ \\
\hline \multicolumn{3}{|l|}{ Situação trabalhista } \\
\hline Autônomo & 1,12 & $(0,54-2,30)$ \\
\hline Celetista & 0,95 & $(0,47-1,95)$ \\
\hline Aposentado/Pensionista & 0,24 & $(0,07-0,74)^{*}$ \\
\hline Desempregado & 0,86 & $(0,34-2,15)$ \\
\hline Outra & 1,00 (Ref.) & - \\
\hline \multicolumn{3}{|l|}{ Possui alguma doença } \\
\hline Sim x Não & 2,89 & $(1,88-4,42)^{*}$ \\
\hline \multicolumn{3}{|c|}{ Última vez que procurou o Serviço de Saúde } \\
\hline Menos de 01 mês & 0,37 & $(0,26-0,52)^{*}$ \\
\hline 01 mês & 0,34 & $(0,23-0,51)^{*}$ \\
\hline 06 meses & 0,46 & $(0,35-0,60)^{*}$ \\
\hline 01 ano & 0,52 & $(0,35-0,75)^{*}$ \\
\hline Mais de 02 anos & 1,00 (Ref.) & - \\
\hline \multicolumn{3}{|l|}{ Motivo da procura } \\
\hline Dor & 3,86 & $(1,10-13,80)^{\prime}$ \\
\hline Doença & 2,87 & $(0,80-10,30)$ \\
\hline Tratamento médico & 1,00 (Ref.) & - \\
\hline Outro & 1,26 & $(0,34-4,65)$ \\
\hline \multicolumn{3}{|l|}{ Serviço de Saúde } \\
\hline Núcleos de Saúde da Família x HER & 0,65 & $(0,50-0,84)^{*}$ \\
\hline
\end{tabular}

${ }^{*} \mathrm{p}<0,05$.

cuidados e prestação de serviços aos outros ${ }^{26}$. Já a relação entre estado civil e procura (variável resposta) não apresentou, neste estudo, nenhuma associação, estando de acordo com pesquisa realizada na Noruega e Finlândia ${ }^{23}$.
A situação econômica é um importante determinante no uso de serviços de saúde, predominando, entre os usuários do SUS, a baixa ren$\mathrm{da}^{27,28}$. Analisando-se os resultados deste estudo, levando-se em consideração que todos os parti- 
Tabela 4. Análises univariadas empregando-se como estimador de associação a Razões de Prevalência (RP) e seu Intervalo de Confiança a 95\% (IC95\%), sendo o sexo dos selecionados a variável resposta. Ribeirão Preto, SP, Brasil.

\begin{tabular}{lcc}
\hline \multicolumn{1}{c}{ Variáveis } & RP & IC 95\% \\
\hline Cidade de Origem (feminino x masculino) & & \\
$\quad$ Ribeirão Preto X Outras & 1,90 & $(1,15-3,12)^{*}$ \\
Faixa Etária (feminino x masculino) & 1,00 (Ref.) & - \\
$\quad$ 18-25 anos & 0,81 & $(0,65-1,00)$ \\
$26-49$ anos & 1,64 & $(1,16-2,05)^{*}$ \\
50-65 anos & & \\
Estado civil (feminino x masculino) & 0,78 & $(0,56-1,08)$ \\
$\quad$ Solteiro & 0,72 & $(0,53-0,90)^{*}$ \\
$\quad$ Casado & $1,00($ Ref.) & - \\
$\quad$ Separado & 0,76 & $(0,50-1,15)$ \\
$\quad$ Viúvo & & $(1,07-1,09)^{*}$ \\
Possui alguma doença (feminino x masculino) & 1,08 & $(0,12-0,76)^{*}$ \\
$\quad$ Não x Sim & & \\
Serviço de Saúde Próximo (feminino x masculino) & 0,31 & \\
$\quad$ Não x Sim & & \\
\hline
\end{tabular}

" $\mathrm{p}<0,05$.

Tabela 5. Resultados da análise multivariada (por regressão logística) ajustada por idade entre os sexos dos fatores positivamente associados à procura por serviços de saúde. Ribeirão Preto/SP, Brasil.

\begin{tabular}{lcc}
\hline \multicolumn{1}{c}{ Variáveis } & RP & IC95\% \\
\hline $\begin{array}{l}\text { Sexo x Procura } \\
\quad \text { Feminino }\end{array}$ & 6,07 & $(3,43-10,76)^{*}$ \\
$\begin{array}{l}\text { Faixa etária } \\
\text { (Feminino x Masculino) } \\
\text { 26-49 anos }\end{array}$ & 3,33 & $(2,09-5,32)^{*}$ \\
\hline
\end{tabular}

${ }^{*} \mathrm{p}<0,05$.

cipantes faziam uso desse tipo de cobertura assistencial, observou-se que entre as famílias que relataram sobreviver mensalmente com um a dois salários mínimos, a prevalência de não procura pelos serviços de saúde foi $61 \%$ menor (RP =0,39; IC95\%: 0,29 - 0,53), quando comparada a famílias com renda maior que essa faixa. Nessa lógica, quando a renda familiar, em comparação à renda individual é menor, devido à divisão salarial per capita, observa-se a renda familiar maior como fator de proteção contra a não procura. De acordo com Pavão et al. ${ }^{29}$, em países como Irlanda e Bélgica, padrões de desigualdade em favor dos mais pobres, considera-se que os aspectos financeiros de isenção de copagamento e redução de taxas para o uso de serviços, provavelmente, tenham incentivado maior uso pela população menos favorecida. Por outro lado, estudo conduzido no Brasil demonstrou achados inversos, apresentando maior uso pelos mais $\operatorname{ricos}^{22}$. Com relação a esse aspecto, Rei e Ramos ${ }^{30}$ indicam que o nível educacional dos trabalhadores parece importante determinante da desigualdade nos rendimentos do trabalho; assim, a renda individual e a familiar foram consideradas como indicador satisfatório de revelação do perfil socioeconômico dos participantes da pesquisa, levando-se em consideração os respectivos graus de escolaridade.

Pode-se, agora, identificar um denominador comum, visto que os resultados das análises efetuadas evidenciaram existência de associação entre procura por serviços de saúde e variáveis ligadas a fatores do ambiente intradomiciliar, ou seja, renda e habitação. Os sujeitos que apresentaram melhores níveis de salário tenderam a ter maiores chances de não procurarem espontaneamente o serviço de saúde, isto é, eles buscaram mais os serviços disponíveis de assistência à saúde. Partindo-se do pressuposto de que há relação intrínseca entre grau de escolaridade e renda, em uma dinâmica em que aqueles que possuem pouca escolaridade são excluídos do mercado de trabalho e substituídos pelos mais capacitados ${ }^{31}$, desencadeando situações que se refletirão na situação trabalhista e nas próprias condições de trabalho, depreende-se que o rendimento condiciona os indivíduos às limitações cotidianas, à 
qualidade e quantidade de bens de aquisição, incluindo vida social, afetando a saúde ${ }^{32}$, havendo então argumento para a associação da maior procura pelos serviços de saúde por aqueles que apresentam menores rendimentos.

Moraes et al..$^{32}$ sugerem que a ocupação é considerada como um dos principais meios de acesso à renda e aos serviços sociais básicos, estando fortemente associada a melhores níveis de saúde. A associação entre situação trabalhista e procura pelos serviços de saúde demonstrou que os aposentados e pensionistas possuem maior prevalência (76\%) por procura aos serviços, demonstrando característica de proteção, vinculando-se ao fato de que a maioria das pessoas aposentadas e pensionistas é idosa, confirmando as percepções já apresentadas sobre o aumento do uso dos serviços de saúde. Isto é o ponto de partida para a discussão sobre associação entre horário de trabalho e procura por assistência à saúde que, neste estudo, não demonstrou estar significativamente associada, não confirmando os achados de outros estudos ${ }^{5}$. Partindo-se da premissa de que os aposentados e pensionistas não desenvolvem mais atividades laborais formais, portanto, limitadas a horários pré-estabelecidos, a amplitude dos horários flexíveis na procura dos serviços de saúde é um fator positivo para a bus$\mathrm{ca}$, enquanto que os trabalhadores sob regime contratual necessitam atender os horários determinados, o que recai sobre o tempo disponível para essa procura. Assim, é de amplo conhecimento que o horário de funcionamento dos serviços de saúde, sobretudo, os da atenção primária não atendem às demandas dos homens, haja vista a coincidência com o turno do horário laboral. Numa análise desse fenômeno, o trabalho lidera a lista das preocupações masculinas, principalmente daqueles que possuem baixa escolaridade, em detrimento da busca pelo serviço de saúde, direcionando sua atenção para o sustento da casa e da família, mais do que para os cuidados com a própria saúde, sugerindo a associação entre ser provedor e ser homem que prevalece na sociedade 5 . O horário de funcionamento dos serviços de saúde é interpretado neste trabalho como barreira institucional. Esta situação costuma encobrir uma dimensão importante do SUS, fazendo-se necessária uma avaliação do fato de que as pessoas que compõem esse sistema, em suas ações, favorecem ou não a construção e a efetivação dos princípios e diretrizes ascendendo sobre o processo saúde-doença. Esse horário, salvo aqueles voltados para atendimentos emergenciais, logo pontuais e não constituidores de vínculos, é incompatível com a disponibilidade da população masculina inserida no mercado formal de trabalho ${ }^{33}$.

Apesar de haver priorização das políticas de atenção voltadas à saúde da mulher, organizando-se os serviços quase que exclusivamente para atender às suas necessidades, não há indissociação no fato contemporâneo de também haver maior participação feminina no mercado de trabalho; no entanto, esse fato não engendrou alteração na estrutura da oferta dos serviços de saúde, talvez devido ao conceito genealógico do cuidado feminino e de sua naturalização e, portanto, considerado, como ascensão social ${ }^{33}$. Ao se criar uma política nacional sobre a saúde do homem, não se reconhecem todas as vertentes dessa questão, situando-a numa polarização cultural, eximindo-se de uma reformulação primordial para que as ações de saúde sejam ofertadas segundo as necessidades do usuário e não do sistema. Se um dos desafios prementes nesse contexto é aumentar a procura e as práticas preventivas, os serviços de saúde devem superar esse desafio de ordem prática. Há, assim, necessidade de melhor acesso e oportunidade de disponibilização dos serviços em tempo adequado, visto que outra característica dessa questão é que nem sempre eles possuem estruturas, recursos humanos e outras condições logísticas para o acesso masculino.

Outra questão foi que, entre aqueles que referiram não possuir nenhuma doença houve prevalência de 2,89 vezes em não procurarem o serviço de saúde regularmente para medidas preventivas. Se, por um lado, as mulheres prevaleceram em relatar alguma doença, por outro, os homens, apesar de não a referirem, possuem um fator de risco de adoecimento, em parte devido ao distanciamento do serviço de saúde, sendo essa uma dicotomia. Os resultados do presente trabalho são coerentes com o padrão universal de que as mulheres fazem mais referências a problemas de saúde do que os homens, e estes não a possuem (subjetivamente), pois, são a representação de virilidade e força, alvos nada fáceis para a fraqueza, ou seja, para a doença ${ }^{26,34}$. Para além desse dado, são notórios os diferenciais de saúde entre os gêneros, tanto por uma medida dele como biológica, sendo o gênero uma das amarras que mais devem ser constituidoras do centro de caracterização e análise da saúde do homem e da mulher ${ }^{8}$, utilizando-se da prerrogativa do aspecto comportamental e da ótica do estilo de vida.

O trabalho de Pires et al. ${ }^{35}$ é convincente ao propor que a organização da oferta dos serviços 
de APS do Distrito Federal sobrecarrega os serviços de média complexidade do local, ao verificar a ocorrência da subutilização da oferta de serviços de saúde, com duplicidade de ações básicas entre os hospitais e unidades básicas de saúde, considerando que poucos conhecem os serviços de saúde do seu município, sobretudo, os centros de saúde $(16,7 \%)$ ou Unidades de Saúde da Família (3,2\%). A leitura dessa categoria demonstra que os moradores do Distrito Federal buscam o hospital por decisão própria, encaminhamentos da unidade básica ou indicação informal, ratificando que as características da demanda por serviços de saúde são determinadas e configuradas pelas características da oferta. Logo, há no entorno do debate a questão de gênero quando atrelada ao fato de os homens não se reconhecerem como alvos do atendimento dos serviços de atenção básica ${ }^{5}$. Em contrapartida, os achados deste estudo não revelaram associações significativas quanto à utilização dos serviços de saúde quando referidos como próximos da residência.

Silva et al. ${ }^{36}$ analisando o perfil de utilização de serviços de saúde no país, demonstraram que ocorreu um decréscimo no número de pessoas que procuraram os serviços para ações de prevenção, aumentando os casos devido a acidentes, lesões e reabilitação. Esse fato está de acordo com os achados deste estudo, visto que o motivo de procura pelo serviço de saúde declarado como "para" prevenção não foi citado, concentrandose em associações de necessidades agudas. Ainda de acordo com os mesmos autores, o motivo da procura na referida pesquisa é de mais de 50\% devido a doenças, seguido daqueles para atividades de prevenção. Adicionalmente, compreendendo que a utilização de rotina ou emergência caracteriza o motivo da procura, verificou-se que, apesar de os estudos ${ }^{36,37}$ demonstrarem a baixa procura para prevenção, a Unidade Básica de Saúde foi o local mais identificado como utilizado. Nesta lógica, considerando o referido serviço como a porta de entrada no sistema, delineando o caminho da descentralização e coordenando a assistência para níveis mais complexos de cuidados, tal apontamento sinaliza que a prestação de serviços via SUS, de acordo com o preconizado legalmente, tem seguido seu fluxo.

$\mathrm{Na}$ análise multivariada, os resultados mostraram que ser do sexo feminino com idade entre 26 a 49 anos foi uma variável associada à maior procura pelo serviço de saúde, em relação aos homens; isso vai ao encontro de estudos conduzidos no Brasil e em outros países, os quais apon- tam o sexo feminino como uma das características demográficas mais associadas ao uso de serviços de saúde ${ }^{23}$. De acordo com Silva ${ }^{33}$ o fato de os homens utilizarem menos os serviços de saúde, acarreta diagnósticos tardios, cujas doenças poderiam ser controladas ou tratadas. Desta forma, homens situados na mesma faixa etária teriam maior predisposição a não procurar os serviços de saúde. No presente trabalho, o recorte de 26 a 49 anos é semelhante ao focado pela Política Nacional de Atenção Integral à Saúde do Homem, a qual infere que essa porção representa $41,3 \%$ da população masculina, sendo a parcela da força produtiva ${ }^{9}$. Assim, ser do sexo feminino com idade entre 26-49 anos apresentaram associações com maior procura dos serviços de saúde, ao passo que ser do sexo masculino, por sua vez, associouse com menor procura.

\section{Conclusão}

Em se tratando de cuidados com a saúde, temse, historicamente na visão de senso comum que o homem é um ser forte, que dificilmente adoece, razão pela qual a procura pelos serviços de saúde apresenta predominância feminina. Como se demonstrou nos resultados das entrevistas, foram identificados diferenciais na procura pelos serviços de saúde, a estrutura social e econômica, que estabelecem diferentes grupos sociais, cujas condições de saúde também são desiguais, e se fazem presentes na procura pelos serviços de saúde, contribuindo para reprodução e acentuação das desigualdades existentes.

Os aspectos culturais, frente aos cuidados com a saúde entre os gêneros ancorados na rede de significados e práticas, foram então retratados para homens e mulheres no quesito participação nos cuidados com a saúde, evidenciando o que se constatou na revisão da literatura. Nessa perspectiva, esses resultados sugerem um grau assertivo de focalização do trabalho dos serviços de saúde nos fatores de risco para a não procura por eles, a saber: ser do sexo masculino, horário de funcionamento dos estabelecimentos de atendimento à saúde versus horário de trabalho do usuário no mercado de trabalho e o fato de terse como resposta não possuir nenhuma doença. Por outro lado, os fatores de proteção contra a não procura, ou seja, de favorecimento da procura - ser usuário dos NSF, ser do sexo feminino e do lar, estar na faixa etária de 26 a 49 anos, possuir domicílio próprio, ter renda familiar $>$ quatro salários mínimos, ser aposentado/pensio- 
nista, comparecer aos retornos, e ser acompanhante de algum familiar ou outros aos serviços de saúde - sinalizam eixos de discussão que necessitam ser aprofundados, de forma a nortear as ações para com os grupos de risco, mediante alternativas que possam construir um processo de trabalho comum e coerente entre usuários e profissionais de saúde.

Busca-se contribuir, assim, para discussão sobre as desigualdades sociais em saúde entre homens e mulheres ao investigar a influência da sociedade, e de todos os determinantes sociais a ela inerentes, sobre a ação de procurar por esses serviços.

\section{Colaboradores}

CD Levorato trabalhou na concepção do projeto, coleta de dados da pesquisa e redação do artigo; LM Mello trabalhou na interpretação dos dados e revisão do artigo; AS Silva trabalhou na interpretação dos dados e revisão do artigo; AA Nunes orientou CD Levorato na condução da pesquisa, análise e interpretação dos resultados e revisão final do artigo. 


\section{Referências}

1. Carloto CM. O conceito de gênero e sua importância para a análise das relações sociais. Serv Soc Rev 2001; 3(2):201-213.

2. Nascimento EF, Gomes R. Marcas identitárias masculinas e a saúde de homens jovens. Cad Saude Publica 2008; 2(7):1556-1564.

3. Schmidt JAC, Anhucci V, Carloto CM. Cidadania: uma discussão sob a perspectiva de gênero. Serv Soc Rev 2005; 8(1):1-8.

4. Villela W. Gênero, saúde dos homens e masculinidades. Cien Saude Colet 2005; 10(1):18-34.

5. Gomes R, Nascimento EF, Araujo FC. Por que os homens buscam menos os serviços de saúde do que as mulheres? As explicações de homens com baixa escolaridade e homens com superior. Cad Saude Publica 2007; 23(3):565-574.

6. Oliffe J.Helth behavior, prostate cancer, and masculinities: a life course perspective. Men Masc 2009; 11(3):346-366

7. Pinheiro RS, Viacava F, Travassos C, Brito AS. Gênero, morbidade, acesso e utilização de serviços de saúde no Brasil. Cien Saude Colet 2002; 7(4):687707.

8. Laurenti R, Jorge MHPDM, Gotlieb SLD. Perfil epidemiológico da morbi-mortalidade masculina. Cien Saude Colet 2005; 10(1):35-46.

9. Brasil. Ministério da Saúde (MS). Secretaria de Atenção à Saúde. Departamento de ações programáticas estratégicas. Política nacional de atenção integral à saúde do homem (Princípios e diretrizes). Brasília (DF): MS; 2008.

10. Brasil. Ministério da Saúde (MS). Instituto Nacional de Câncer (INCA). Estimativa 2010 - Incidência de câncer no Brasil. [acessado 2011 jan 12]. Disponível em: http://www.inca.gov.br/estimativa/2010/ estimativa20091201.pdf

11. Brasil. Ministério da Saúde (MS). Portal da Saúde. Profissional e Gestor. Pesquisa Sociedade Brasileira de Urologia (SBU). Homens não fazem exame de toque, mesmo sabendo que teste é usado para detectar o câncer. [Internet]. [acessado 2011 jan 11]. Disponível em: http://portal.saude.gov.br/portal/saude/ visualizar_texto.cfm?idtxt $=34191$ \&janela $=1$

12. Atenção à saúde do homem. Revista Pesquisa Médica 2008; 8 out/dez. (on line). [acessado 2012 ago 20]. Disponível em: http://www.revistapesquisamedica. com.br/portal/textos.asp?codigo $=11432$

13. Brasil. Ministério da Saúde (MS). Secretaria de Vigilância em Saúde. Departamento de Análise de Situação em Saúde. Série G. Estatística e Informação em Saúde. Saúde Brasil 2007: uma análise da situação de saúde. Mortalidade por uso de álcool. Brasília (DF): Ministério da Saúde. [acessado 2011 jan 11] Disponível em: http://portal.saude.gov.br/portal/arquivos/pdf/saude_brasil_2007.pdf.

14. Brasil. Ministério da Saúde (MS). Secretaria de Vigilância em Saúde. Departamento de Análise de Situação em Saúde. Série G. Estatística e Informação em Saúde. Saúde Brasil 2007 - Uma análise da situação de saúde. A violência no Brasil: abordando diferentes pontos. Brasília (DF): MS. [acessado 2011 jan 11] Disponível em: http://portal.saude.gov.br/ portal/arquivos/pdf/saude_brasil_2007.pdf
15. Brasil. Ministério da Saúde (MS). Secretaria de Vigilância em Saúde. Secretaria de Gestão Estratégica e Participativa. Vigitel Brasil 2008: vigilância de fatores de risco e proteção para doenças crônicas por inquérito telefônico. Estimativas para 2008 - Tabagismo. Brasília (DF): MS. [acessado 2011 jan 11]. Disponível em: http://portal.saude.gov.br/portal/arquivos/ pdf/VIGITEL2008_web.pdf

16. Brasil. Ministério da Saúde (MS). Secretaria de Vigilância em Saúde. Secretaria de Gestão Estratégica e Participativa. Vigitel Brasil 2008: vigilância de fatores de risco e proteção para doenças crônicas por inquérito telefônico. Estimativas para 2008 - Excesso de peso e obesidade. Brasília: MS; 2008:34-9. [acessado 2011 jan 11]. Disponível em: http://portal.saude. gov.br/portal/arquivos/pdf/VIGITEL2008_web.pdf

17. Brasil. Ministério da Saúde (MS). Secretaria de Vigilância em Saúde. Pesquisa de conhecimentos, atitudes e práticas relacionada à DST e Aids da população brasileira de 15 a 64 anos de idade. 2008. [acessado 2011 jan 12]. Disponível em: http://www.aids. gov.br/sites/default/files/PCAP-BR_CNAIDS.ppt

18. Nunes AA, Caccia-Bava MCGG, Cisoto RL, Pereira MJB, Santos V, Watanabe M, Domingues N, Grupo Pet-Saúde USP/RP2009. Resolubilidade da Estratégia Saúde da Família e Unidades Básicas de Saúde tradicionais: contribuições do PET-Saúde. Rev Bras Educ Med 2012; 36(1):27-32.

19. Brasil. Conselho Nacional de Secretários de Saúde. Atenção Primária e Promoção da Saúde. Coleção Progestores - Para entender a Gestão do SUS. V. 8. [Internet] 2007 [acessado 2011 set 11]. Disponível em: http://bvsms.saude.gov.br/bvs/publicacoes/ colec_progestores_livro8.pdf

20. Ribeiro MM. Utilização de Serviços de Saúde no Brasil: uma investigação do padrão etário por sexo e cobertura por plano de saúde [dissertação]. Belo Horizonte: Universidade Federal de Minas Gerais; 2005.

21. Brasil. Instituto Brasileiro de Geografia e Estatística (IBGE). Síntese de indicadores sociais: uma análise das condições de vida da população brasileira; 2010. Rio de Janeiro: IBGE; 2010. [acessado 2011 out 8]. Disponível em: http://www.ibge.gov.br/home/estatistica/populacao/condicaodevida/indicadoresminimos/sinteseindicsociais2010/SIS_2010.pdf.

22. Travassos C, Viacava F, Pinheiro R, Brito, A. Utilização dos serviços de saúde no Brasil: gênero, características familiares e condição social. $\mathrm{Rev} \mathrm{Pa}$ nam Salud Publica 2002; 11(5/6):365-373.

23. Lima-Costa MF, Loyola Filho AI. Fatores associados ao uso e à satisfação com os serviços de saúde entre usuários do Sistema Único de Saúde na região metropolitana de Belo Horizonte, MG, Brasil. Epidemiol Serv Saude 2008; 17(4):247-257.

24. Costa MFL. Influência da idade e da escolaridade no uso de serviços preventivos de saúde - Inquérito de Saúde da Região Metropolitana de Belo Horizonte, MG, Brasil. Epidemiol Serv Saude 2004; 13(4):209-215.

25. Mendes TAB. Prevalência de doenças crônicas e utilização de serviços de saúde por idosos residentes no município de São Paulo [tese]. São Paulo: Universidade de São Paulo; 2010 
26. Lyra J, Leão LS, Lima DC, Targino P, Crisóstomo A, Santos B. Homens e cuidado: uma outra família? $3^{a}$ ed. São Paulo: Cortez; 2008.

27. Brasil. Instituto Brasileiro de Geografia e Estatística (IBGE). Pesquisa Nacional por Amostra de Domicílios (PNAD). Síntese de indicadores, 2009. Rio de Janeiro. 2010. [acessado 2011 out 8]. Disponível em: http://www.ibge.gov.br/home/estatistica/populacao/ trabalhoerendimento/pnad2009/pnad_sintese_ 2009.pdf

28. Lima-Costa MF, Matos DL, Camarano AA. Evolução das desigualdades sociais em saúde entre idosos e adultos brasileiros: um estudo baseado na Pesquisa Nacional por Amostra de Domicílios (PNAD 1998, 2003). Cien Saude Colet 2006; 11(4):941-950.

29. Pavão ALB, Coeli CM, Lopes CS, Werneck GL, Chor D. Uso de serviços de saúde segundo posição socioeconômica em trabalhadores de uma universidade pública. Rev Saude Publica 2012; 46(1):98-103.

30. Reis MC, Ramos L. Escolaridade dos pais, desempenho no mercado de trabalho e desigualdade de rendimentos. Rev Bras Econ 2011; 65(2):177-205.

31. Chahad JPZ. Tendências recentes no mercado de trabalho: pesquisa de emprego e desemprego. São Paulo Perspec 2003; 17(3-4):205-217.

32. Moraes JR, Moreira JPL, Luiz RR. Associação entre o estado de saúde autorreferido de adultos e a área de localização do domicílio: uma análise de regressão logística ordinal usando a PNAD 2008. Cien Saude Colet 2011; 16(9):3769-3780.

33. Silva VLQ. Sexualidade masculina e saúde do homem na Estratégia Saúde da Família: trabalhando com a equipe a pesquisa-ação [dissertação]. Ribeirão Preto: Universidade de São Paulo; 2009.

34. Viacava F. Acesso e uso de serviços de saúde pelos brasileiros. Radis comunicação em saúde 2010; 96:1219.

35. Pires MRGM, Göttems LBD, Martins CMF, Guilhem D, Alves ED. Oferta e demanda por média complexidade/SUS: relação com atenção básica. Cien Saude Colet 2010; 15(Supl. 1):1009-1019.

36. Silva ZP, Ribeiro MCSA, Barata RB, Almeida MC. Perfil sociodemográfico e padrão de utilização dos serviços de saúde do Sistema Único de Saúde (SUS), 2003-2008. Cien Saude Colet 2011; 16(9):3807-3816.

37. Victoria CG, Barreto ML, Leal MC, Monteiro CA, Schmidt MI, Paim J, Bastos FI, Almeida C, Bahia L, Travassos C, Reichenheim M, Barros FC, the Lancet Brazil Series Working Group. Condições de saúde e inovações nas políticas de saúde no Brasil: o caminho a percorrer. Lancet 2011; 6(Supl. Saúde no Brasil):90-102.

Artigo apresentado em 18/03/2013

Aprovado em 20/04/2013

Versão final apresentada em 29/04/2013 